\title{
Avaliação do impacto do tempo de espera para admissão em Unidade de Terapia Intensiva no desfecho clínico do paciente crítico
}

\author{
Deorgelis Rosso ${ }^{1}$ (D), Glauco Adrieno Westphal ${ }^{2}$ (D), Larissa Fabre ${ }^{3}$ (D), Maria Luiza Floriano ${ }^{4}$ (D),
} Mariana Laís Mendes ${ }^{4}$ (D) Morgana Longo 3 (D) Patricia Tessari ${ }^{4}$ (D)

\begin{abstract}
RESUMO
Este estudo tem por base a premissa de que com um maior número de leitos de Unidade de Terapia Intensiva (UTI) disponíveis o tempo de espera para admissão em UTI é menor, o que resulta no melhor desfecho clínico, justificando, portanto, a importância do presente estudo. Objetivo: Avaliar se o tempo de espera no Departamento de Emergência até a admissão em UTI tem influência no desfecho clínico do paciente crítico. Metodologia: Estudo observacional, retrospectivo, do tipo antes e depois, realizado em um hospital público do município de Joinville/SC no ano de 2019. Foram incluídos os dados referentes aos pacientes adultos admitidos na UTI com até 72 horas de espera no Departamento de Emergência desde a chegada ao hospital. Comparou-se o último trimestre de 2017 (fase 1), período durante o qual havia 14 leitos de UTI no hospital, e último trimestre de 2018 (fase 2), período durante o qual havia 30 leitos de UTI. Resultados: Analisaram-se 173 prontuários elegíveis de 2017 e 2018. Houve diferença estatisticamente significativa no tempo decorrido na emergência até a admissão em UTI entre 2017 e 2018 (mediana de 22 vs. $15 ; p=0,0002$ ). A diferença estatística também foi relevante para a mortalidade em até 24 horas após a admissão em UTI, comparando-se os dois anos em questão $(9,61 \%$ vs. $2,47 \% ; p=0,04)$. Não houve diferença estatística significante na mortalidade hospitalar entre 2017 e 2018 (34,6\% vs. 35,5\%; p=0,57). Também não houve diferença estatisticamente relevante entre os demais parâmetros analisados. Conclusão: Comparando-se 2017 a 2018, percebeu-se que o tempo de espera pelo leito de UTI diminuiu, bem como a mortalidade em até $24 \mathrm{~h}$ da admissão intensiva. No entanto, isto não se refletiu na mortalidade hospitalar.
\end{abstract}

Palavras-chave: Emergências; Unidades de Terapia Intensiva; Mortalidade.

\section{INTRODUÇÃO}

A demanda por cuidados intensivos está aumentando em todo o mundo, porém, há grandes variações regionais na disponibilidade de leitos nas Unidades de Terapia Intensiva (UTI) ${ }^{1}$. No Brasil, segundo dados de 2020 do Ministério da Saúde, há 23 mil leitos de UTI adulto e pediátrico para atender a população². Entretanto, devido ao aumento progressivo na chegada de pacientes críticos nos setores de emergência, cenários de superlotação e o caráter de funcionamento agitado destes setores, médicos e enfermeiros enfrentam dificuldades para assistir aos pacientes críticos da forma adequada ${ }^{3}$. Esta realidade dos setores de emergência resulta diretamente em atrasos na transferência destes pacientes críticos aos leitos de UTI, uma vez que os médicos demoram mais a serem notificados de progressivas deteriorações no estado de saúde do paciente, bem como demoram mais para avaliar os mesmos nestes ambientes superlotados dos setores de emergência 4 .

Neste contexto, estudos sugerem que um tempo de espera maior para a transferência até um leito de UTI repercuta drasticamente no desfecho do paciente. Um estudo de coorte realizado no hospital McKay-Dee em Ogden, Utah, em 2003, observou que a demora na admissão dos pacientes na UTI resultou em aumento da mortalidade, aumento dos custos com recursos hospitalares e maior morbidade quando comparado ao grupo com tempo de admissão em UTI menor de 4 horas. Esses piores desfechos foram associados a atrasos na notificação da deterioração clínica ao médico e atraso também

Médico intensivista do Hospital Municipal São José (HMSJ) - Orientador. Joinville (SC), Brasil.

Residente de Clínica Médica do Hospital Municipal São José (HMSJ) - Autora principal. Joinville (SC), Brasil.

Acadêmica do quinto ano de Medicina, Universidade da Região de Joinville (UNIVILLE) - Autora adjunta. Joinville (SC), Brasil. 
na avaliação médica à beira do leito, sugerindo que os pacientes de transferência lenta receberam tratamento médico diferente daqueles com transferência rápida ${ }^{4}$. De modo semelhante, um estudo transversal de 2007 mostrou que, entre 2000 e 2003, pacientes com 6 horas ou mais de espera para o leito de UTI tiveram maior tempo de internação, maior mortalidade na UTI e maior mortalidade intra-hospitalar em geral ${ }^{3}$. Além disso, intervenções como ventilação mecânica e acesso venoso central foram mais utilizados no grupo com mais de 6 horas até a admissão em $\mathrm{UTI}^{3}$. Sabe-se que o impacto positivo da admissão em UTI na sobrevida do paciente é mais evidente durante as primeiras 72 horas de doença crítica ${ }^{5}$.

A admissão tardia na UTI pode estar relacionada não somente à indisponibilidade de leitos, mas também à falta de protocolos hospitalares de transferência interna que auxiliem no manejo de pacientes críticos $^{3}$. Ainda, em um estudo realizado em 2003, os desfechos desfavoráveis encontrados no grupo com tempo para admissão em UTI maior que 4 horas foram a demora na notificação do médico a respeito da piora clínica do paciente e a demora maior que 3 horas para avaliação médica do paciente a partir de alcançado o primeiro critério fisiológico de gravidade, mesmo quando há notificação imediata ao médico ${ }^{4}$.

Dentro deste contexto, foi realizado o presente estudo com o objetivo principal de avaliar se o tempo de espera no Departamento de Emergência, desde a chegada do paciente ao hospital, até o ingresso na UTI influência nos desfechos de mortalidade hospitalar e mortalidade em até 24 horas após admissão em UTI.

\section{MATERIAL E MÉTODOS}

Estudo observacional, retrospectivo, do tipo antes e depois, realizado em um hospital público do município de Joinville, em Santa Catarina, no ano de 2019. Foram analisados dados referentes aos pacientes adultos admitidos na UTI no último trimestre de 2017 (fase 1), período durante o qual havia 14 leitos de UTI no hospital, e no último trimestre de 2018 (fase 2), período durante o qual havia 30 leitos de UTI no hospital. Este projeto foi aprovado pelo Comitê de Ética sob o número 05922819000005362, respeitando as resoluções 466/12.
Foram avaliados todos os pacientes admitidos no hospital e provenientes diretamente do Departamento de Emergência. Como requisito para inclusão nesta pesquisa, foi determinado que o tempo máximo de permanência no setor de emergência, desde a chegada dos pacientes ao hospital até a admissão em UTI, seria de até 72 horas. Foram excluídos do estudo, portanto, os pacientes menores de 18 anos, aqueles em pós-operatório de cirurgias eletivas, os pacientes submetidos à transferência inter-hospitalar ou provenientes de outros setores do hospital e aqueles com mais de $72 \mathrm{~h}$ desde a admissão hospitalar até a admissão em UTI. Admissão tardia foi considerada como sendo o tempo superior a seis horas a partir do momento da admissão do paciente no serviço de emergência do hospital até sua admissão na UTI.

Foram coletados os seguintes dados de cada paciente: idade, sexo, diagnóstico de admissão na UTI, Simplified Acute Physiology Score 3 (SAPS3) e tempo de espera pelo leito de UTI ${ }^{6}$. As variáveis de desfecho analisadas foram: mortalidade hospitalar e mortalidade em até 24 horas após a admissão na UTI.

O SAPS 3 constitui-se de um sistema com 20 variáveis que devem ser observadas na admissão do paciente na UTI. Através do preenchimento desta escala, que tem os valores entre 16 e 217 , é possível avaliar a condição clínica do paciente previamente à internação, além de explanar a extensão do comprometimento do estado de saúde do mesmo. Para tanto, avaliam-se variáveis fisiológicas, variáveis demográficas e razão para admissão em UTI ${ }^{6}$.

Variáveis contínuas foram analisadas utilizando o teste $T$ de Student e teste $U$ de MannWhitney, conforme apropriado. Variáveis categóricas foram analisadas utilizando o teste qui-quadrado. Modelos de regressão logística foram utilizados para analisar as variáveis associadas à mortalidade de maneira independente. Os dados foram registrados no Microsoft Excel 2013 (Microsoft, Redmond, Washington, EUA) e analisados através do Statistical Package for Social Sciences (SPSS), versão 20.0 (IBM Corp, NY, Estados Unidos).

\section{RESULTADOS}

Durante o período do estudo, ocorreram 381 admissões na UTI. Um total de 208 (54,5\%) 
pacientes foram excluídos do estudo devido aos seguintes fatores: $47(22,5 \%)$ pacientes eram pós-operatório de cirurgia eletiva, $6(2,8 \%)$ eram menores de 18 anos, $15(7,2 \%)$ eram provenientes de outros setores do hospital, $121(58,1 \%)$ foram admitidos após 72 horas da admissão hospitalar e $19(9,1 \%)$ vieram transferidos de outro hospital. No total foram analisados os dados de 52 (30\%) pacientes em 2017 e 121 (69,9\%) pacientes em 2018 (Figura 1).

Com relação às características da amostra, a mediana da idade dos pacientes foi de 51 (IQR 42-59) e 55 anos (IQR 34-65) em 2017 e 2018, respectivamente, com predomínio do sexo masculino em ambos os anos (59,6\% e 61,1\%) (Tabela 1). A frequência de admissões tardias na UTI foi de $81,5 \%$, sendo que em 2017 essa taxa foi de 96,1\% e em 2018 foi de 75,2\% (Tabela 1). Os valores da mediana do SAPS3 encontrados foram de 56 (IQR 43-66) em
2017 e 66 (IQR 51-80) em 2018 (Tabela 1). Ainda, houve diferença estatisticamente significativa entre os grupos de 2017 e 2018 quanto ao tempo em horas para a admissão na UTI (mediana de 22, IQR 18-47 vs. 15 , IQR 7-30; $p=0,0002)$.

Quanto aos desfechos (Tabela 2), houve diferença estatisticamente significativa para a mortalidade em até 24 horas após a admissão na UTI $(9,61 \%$ vs. $2,47 \% ; p=0,040)$. Com relação à mortalidade hospitalar, não houve diferença estatística significante quando comparados os dois anos (34,6\% vs. $35,5 \% ; p=0,570)$.

Com base na análise de regressão logística binária, não houve associação entre o tempo de espera em horas para a admissão na UTI e a mortalidade hospitalar (OR 1,001, IC 95\% [0,982 a $1,021], p=0,886$ ) (Tabela 3) ou a mortalidade em até 24 horas após a admissão na UTI (OR 0,943, IC $95 \%[0,886$ a 1,004], $p=0,068$ ) (Tabela 4).

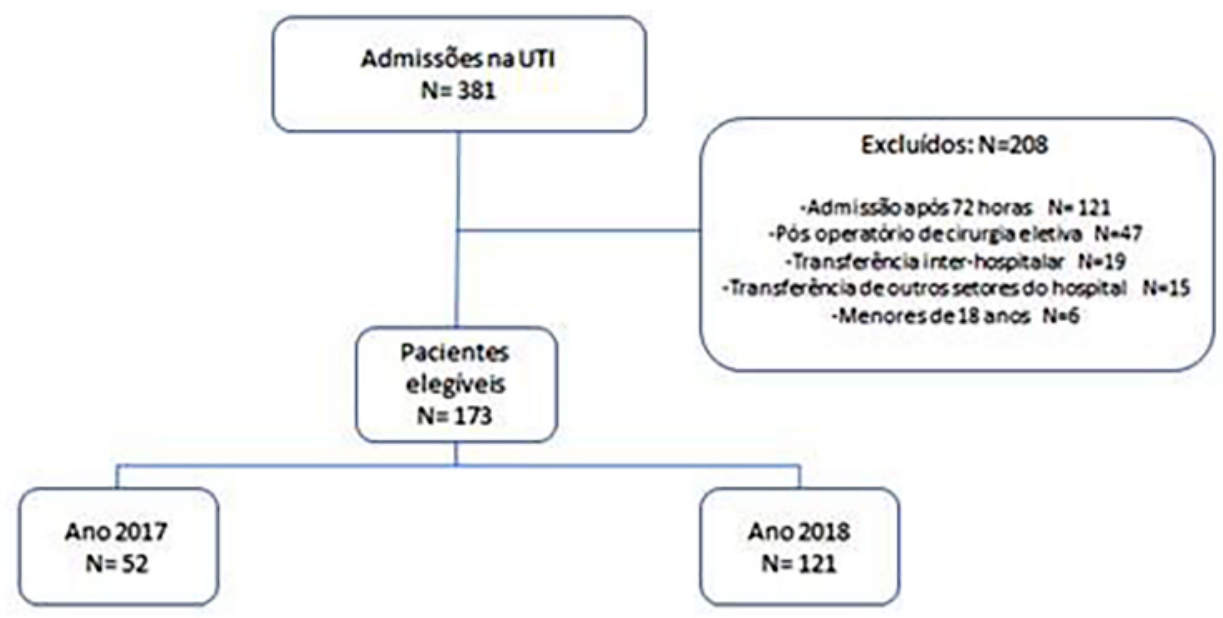

Figura 1: Fluxograma de pacientes admitidos na Unidade de Terapia Intensiva no último trimestre de 2017 e 2018.

Não houve associação entre admissão tardia e mortalidade hospitalar (OR 1,20, IC 95\% [0,475 a 3,071], $p=0,69$ ) (Tabela 3). Esta associação também não ocorreu entre a admissão tardia e a mortalidade em até 24 horas após a admissão na UTI (OR 0,136, IC 95\% [0,010 a 1,800], P=0,13) (Tabela 4).
Com relação ao aumento nos valores do SAPS 3 , estes foram sim, associados a taxas de mortalidade mais elevadas nas primeiras 24 horas de admissão na UTI (OR 1,129, IC 95\% [1,043 a 1,222], p= $0,003)$ (Tabela 4), porém não se associaram a maiores taxas de mortalidade hospitalar (OR 1,002, IC 95\% [0,989 a 1,014], $\mathrm{p}=0,788$ ) (Tabela 3). 
Tabela 1

Características clínicas e demográficas dos pacientes admitidos na UTI em 2017 e 2018.

\begin{tabular}{lccc}
\hline Características do paciente & $\mathbf{2 0 1 7}(\mathbf{n = 5 2})$ & $\mathbf{2 0 1 8}(\mathbf{n = 1 2 1})$ & Valor de P \\
\hline Masculino, $\mathbf{n}(\%)$ & $31(59,6)$ & $74(61,1)$ & 0,85 \\
Idade, anos, mediana (IQR) & $51(42-59)$ & $55(34-65)$ & 0,44 \\
SAPS 3, mediana (IQR) & $56(43-66)$ & $66(51-80)$ & 0,001 \\
Tempo de espera para admissão na & $22(18-47)$ & $15(7-30)$ & 0,0002 \\
UTI, horas, mediana (IQR) & $50(96,15 \%)$ & $91(75,2 \%)$ & 0,0011 \\
Admissão tardia, $\mathbf{n}(\%)$ & & & \\
\hline
\end{tabular}

Tabela 2

Desfechos das admissões na Unidade de Terapia de Intensiva em 2017 e 2018

\begin{tabular}{lccc}
\hline \multicolumn{1}{c}{ Desfechos } & $\mathbf{2 0 1 7}(\mathbf{n = 5 2 )}$ & $\mathbf{2 0 1 8}(\mathbf{n = 1 2 1})$ & Valor de $\mathbf{P}$ \\
\hline $\begin{array}{l}\text { Mortalidade hospitalar, } \mathbf{n}(\%) \\
\text { Mortalidade hospitalar em até } \mathbf{2 4} \mathbf{~ h}\end{array}$ & $18(34,6)$ & $43(35,5)$ & 0,57 \\
após admissão na UTI, $\mathbf{n ~ ( \% )}$ & $5(9,61)$ & $3(2,47)$ & 0,04 \\
\hline
\end{tabular}

Tabela 3

Análise da regressão binária - variável dependente sendo a mortalidade hospitalar, n

\begin{tabular}{lccc}
\hline \multicolumn{1}{c}{ Covariáveis } & Odds ratio & $\mathbf{9 5 \%}$ IC & Valor de P \\
\hline Idade & 1,015 & $0,996-1,035$ & 0,131 \\
Sexo masculino & 0,710 & $0,371-1,357$ & 0,300 \\
SAPS 3 & 1,002 & $0,989-1,014$ & 0,788 \\
Admissão tardia & 1,207 & $0,475-3,071$ & 0,692 \\
Tempo de espera para admissão na & 1,001 & $0,982-1,021$ & 0,886 \\
UTI & & & \\
\hline
\end{tabular}

Tabela 4

Análise da regressão binária - variável dependente sendo a mortalidade hospitalar em até 24 horas após a admissão na UTI, $\mathrm{n}$

\begin{tabular}{lccc}
\hline \multicolumn{1}{c}{ Covariáveis } & Odds ratio & $\mathbf{9 5 \%}$ IC & Valor de P \\
\hline Idade & 0,955 & $0,910-1,003$ & 0,065 \\
Sexo masculino & 1,297 & $0,242-6,942$ & 0,761 \\
SAPS 3 & 1,129 & $1,043-1,222$ & 0,003 \\
Admissão tardia & 0,136 & $0,010-1,800$ & 0,130 \\
Tempo de espera para admissão na UTI & 0,943 & $0,886-1,004$ & 0,068 \\
\hline
\end{tabular}

\section{DISCUSSÃO}

Em 2018 foi observado menor tempo de espera para admissão em UTI (mediana em horas de 22 em 2017 vs. 15 em 2018, $p=0,0002$ ). Entende-se essa mudança como fruto da ampliação de leitos de UTI ocorrido no segundo ano analisado. Como resultado desta modificação, foi observado também o menor índice de admissões tardias neste ano (96,1\% em 2017 vs. 75,2\% em 2018). No entanto, a frequência geral de admissões tardias no presente artigo foi de $81,5 \%$, considerada ainda bastante elevada quando comparada aos dados da literatura sobre admissões tardias, por exemplo, em regiões como Israel (24\%), França $(37,6 \%)$, Inglaterra $(32,6 \%)$ e Hong Kong $(37,8 \%)^{7-10}$.

Estudo observacional retrospectivo em um hospital universitário de Boston (EUA) com 287 pacientes com sepse grave ou choque séptico, categorizou retardo de internação em UTI como aquela que ocorre após um tempo decorrido de 6h. Neste mesmo estudo, encontrou-se uma taxa de 52,2\% de transferências à UTI com mais de $6 \mathrm{~h}$ de permanência na emergência. Todos os pacientes passaram 
por protocolo rígido de tratamento e ressuscitação de sepse. Não foram encontradas diferenças significativas nos desfechos clínicos dos dois grupos, provavelmente imagina-se que isto ocorreu devido aos cuidados intensos e especializados disponibilizados ao paciente já no setor de emergência deste hospital universitário. Isto reforça a tese de que "cuidados de UTI" podem e devem ser iniciados ainda nos setores de emergência, nas primeiras horas de atendimento, até que se disponibilize o acesso ao leito especializado ${ }^{11}$.

Assim, as admissões tardias no presente estudo refletem também a sobrecarga de trabalho no setor de emergência, o que alerta para a necessidade de melhorias no processo de admissão e transferência hospitalar interna de pacientes críticos. Como fundamentação disto, uma análise prospectiva de um artigo com pesquisa realizada em 81 pacientes demonstrou que embora o tempo de permanência na emergência seja muito reduzido quando comparado ao período de internação hospitalar e em UTI, os cuidados dispensados aos pacientes críticos nessa fase de permanência nos setores de emergência impactam significativamente na redução da progressão das disfunções orgânicas e melhora da sobrevida ${ }^{12}$.

Considera-se que estes cuidados nos setores de emergência sejam essenciais à instituição deste presente estudo. Visto que, mesmo com número pequeno de pacientes, foi possível observar a associação entre o aumento nos valores do SAPS3 e a elevação das taxas de mortalidade nas primeiras 24 horas de admissão em UTI (OR 1,129, IC 95\% [1,043 a 1,222$], p=0,003)$. Deste modo, a estabilização do paciente para admissão em UTI e a transferência precoce, que depende essencialmente do manejo inicial nos setores de emergência, contribuiria para redução dos valores de SAPS3 e, portanto, para o melhor desfecho clínico nas primeiras 24 horas.

Entretanto, sabe-se das dificuldades em relação à indisponibilidade de equipamentos e insumos para realizar uma assistência completa nos setores de emergência e da escassez de leitos especializados. Conforme dados do censo de 2016 da Associação de Medicina Intensiva Brasileira, no Brasil apenas 15\% dos municípios apresentam leitos de UTI ${ }^{13}$. Conforme recomendações da Organização Mundial de Saúde e do Ministério da Saúde, a quantidade ideal de leitos de UTI é de 1 a 3 leitos para cada 10 mil habitantes, sendo que o Brasil apresenta a proporção de 2,2 leitos, o que, de forma isolada, parece satisfatório. Entretanto, existe uma discrepância importante entre o setor público e o privado: o SUS possui em média 1,4 leitos para cada 10 mil habitantes, enquanto o setor privado conta com uma média de $4,9^{14}$.

O hospital público no qual este estudo foi realizado é referência estadual em transplantes, traumatologia, neurologia, oncologia e tratamento de queimados. Portanto, apresenta um alto fluxo de paciente de diversas cidades do estado, em condições muitas vezes críticas e que necessitam de internação em UTI, fator que motivou o acréscimo dos leitos de UTI em 2018. De modo semelhante, a maioria dos hospitais públicos do Brasil enfrenta taxas de ocupação elevadas, especialmente em hospitais de grande porte $^{15}$. Quando a demanda supera a oferta, o acesso se torna limitado e o atendimento dos pacientes é postergado. Ultrapassando as fronteiras, um estudo realizado em três hospitais da Austrália em 2006, já encontrou um aumento relativo de $30 \%$ da mortalidade no segundo e no sétimo dia em hospitais com superlotação, inclusive nos setores de emergência, para pacientes que necessitam de internação ${ }^{16}$. Além disso, independentemente da estrutura física, a qualificação da equipe, a formulação de protocolos de atendimento bem definidos e a experiência no manejo de pacientes graves pelos setores de emergência é determinante para a aprimorar o desfecho clínico após a entrada na terapia intensiva.

Com relação ao SAPS3, encontrou-se associação deste com a mortalidade em até 24 horas após a admissão em UTI (OD 1,129, IC 95\% [1,043 a $1,222], p=0,003)$, porém a associação com a mortalidade hospitalar não foi encontrada (OD 1,002, IC $95 \%[0,989$ a 1,014$], p=0,788)$. Observou-se que em 2018 o valor da mediana do SAPS3 mostrou-se maior (66 em 2018 vs. 56 em 2017), apontando para admissão de pacientes em estado mais grave na UTI. No entanto, mesmo diante deste cenário, ainda houve diminuição da mortalidade em 24 horas da admissão em UTI quando comparado 2018 a 2017. Portanto, questiona-se se o menor tempo de espera para admissão (mediana de 22 horas em 2017 vs. 15 horas em 2018) e a menor taxa de admissão tardia em 2018 (96,15\% em 2017 vs. 75,2\% em 2018) poderiam ser responsáveis pela diminuição da mortalidade em 24 horas observada em 2018.

Com relação à admissão tardia relacionada a mortalidade hospitalar, em 2005 foi realizada 
uma coorte prospectiva no Hospital Universitário de Londrina que avaliou 401 pacientes provenientes do próprio hospital para a UTI, categorizando-os em admissão atrasada ou não, com corte máximo de 72 horas. Destes, 276 pacientes tiveram retardo de acesso à UTI $(>72 \mathrm{~h})$, resultando em uma mortalidade de $138(50 \%)$, versus $47(37,6 \%)$ dos internados sem atraso em UTI. Os pacientes de permanência prolongada no setor de emergência tiveram maior chance de complicação com quadro de sepse: 172 casos $(62,3 \%)$ em admissões tardias e 58 casos $(46,8 \%)$ em imediatas 5 .

Assim como o estudo citado, este presente estudo buscou relacionar os desfechos clínicos dos pacientes com a demora de admissão de leito em UTI utilizando um corte máximo de 72 horas. Portanto, pacientes admitidos após mais de 72 horas de espera no setor de emergência foram excluídos. Este tempo de corte foi determinado, pois é o limite a partir do qual se excederia a janela de benefício nestes pacientes críticos, já que são nas primeiras 72 horas da evolução de um quadro grave que os maiores resultados do cuidado intensivo transparecem clinicamente. ${ }^{5}$ Entretanto, os dados finais não conseguiram demonstrar aumento da mortalidade hospitalar nos casos de admissão tardia, não havendo diferença estatisticamente significante entre estes parâmetros (OD 1,207, IC $95 \%$ [0,475 a 3,071], $p=0,692)$. Também não houve diferença estatisticamente significante na mortalidade hospitalar entre 2017 e $2018(p=0,57)$.

Isto ocorreu mesmo com a maior demora para admissão em UTI em 2017 (mediana de 22 em 2017 vs. 15 em 2018). Discute-se se o menor tempo de espera em 2018 ocorreu em virtude do aumento do número de leitos neste ano, impactando na diminuição da mortalidade em 24 horas de admissão na UTI neste ano. Porém, em virtude do número pequeno de pacientes no presente estudo, o impacto clínico destas intervenções pode não ter sido suficientemente expressivo no que tange à mortalidade hospitalar.

Ao analisar os dados do estudo, percebe-se que um dos maiores determinantes para o desfecho do paciente crítico seria a intervenção imediata de suporte aos órgãos, que é iniciada na sala de emergência. Este conceito de "cuidado crítico sem paredes" vem se tornando cada vez mais aceito na atualidade.

As principais limitações desse estudo foram o fato de ser monocêntrico e o pequeno tamanho da amostra.

\section{CONCLUSÃO}

Constatou-se, portanto, que com aumento do número de leitos de UTI disponíveis de 2017 para 2018 houve uma diminuição do tempo de espera por esses leitos e menor índice de admissões tardias, acompanhadas da redução na mortalidade em até $24 \mathrm{~h}$, o que aponta para melhor desfecho precoce quando há admissão em tempo adequado em UTI e a capacidade da equipe em manter uma boa assistência inicial ao paciente mesmo com maior número de leitos.

No entanto, estes achados não se refletiram na mortalidade hospitalar, uma vez que não houve diferença estatisticamente relevante deste parâmetro entre os anos estudados. Isto levanta questionamentos no que tange ao número de leitos de UTI serem constantemente considerados, por gestores e até pela população em geral, de forma isolada, como único parâmetro preditor de desfecho dos pacientes críticos. Além disso, o tempo de espera no departamento de emergência para a admissão na UTI não demonstrou associação com as taxas de mortalidade neste estudo. Porém, cabe salientar que, caso fossem avaliados outros desfechos e reunido um maior número de pacientes, a associação entre o tempo de espera pelo leito de UTI e a mortalidade hospitalar em pacientes graves pudesse ser encontrada.

\section{REFERÊNCIAS}

1. Wild C, Narath M. Evaluating and planning ICUs: methods and approaches to differentiate between need and demand. Health Policy. 2005; 71(3):289-301.

2. Ministério da Saúde (BR). Ministério da Saúde autoriza abertura de 1,4 mil leitos de UTI em todo o País. Saúde e vigilância sanitária. Jan 2020. Disponível em: https:// bit.ly/3wHMvZt

3. Chalfin DB, Trzeciak S, Likourezos A, Baumann BM, Dellinger RP. Impact of delayed transfer of critically ill patients from the emergency department to the intensive care unit. Crit Care Med. 2007; 35(6):1477-83.

4. Young MP, Gooder VJ, McBride K, James B, Fisher ES. Inpatient transfers to the intensive care unit: delays are associated with increased mortality and morbidity. J Gen Intern Med. 2003; 18(2):77-83.

5. Cardoso LT, Grion CM, Matsuo T, Anami EH, Amkauss I, Seko $L$, et al. Impact of delayed admission to intensive care units on mortality of critically ill patients: a cohort study. Critical Care. 2011; 15 (1): 28-36.

6. Silva Junior JM, Malbouisson LMS, Nuevo HL, Barbosa LGT, Marubayashi LY, Teixeira IC, et al. Aplicabilidade do escore 
fisiológico agudo simplificado (SAPS 3) em hospitais brasileiros. Rev. Bras. Anestesiol. 2010; 60(1): 20-31.

7. Sprung $C L$, Geber $D$, Eidelman $L A$, Baras $M$, Pizov $R$, Nimrod $A$, et al. Evaluation of triage decisions for intensive care admission. Crit Care Med. 1999; 27(6):1073-79.

8. Garrouste-Orgeas M, Montuclard L, Timsit JF, Reignier J, Desmettre T, Karoubi $P$, et al. Predictors of intensive care unit refusal in French intensive care units: A multiplecenter study. Crit Care Med.2005; 33(4):750-5.

9. Metcalfe MA, Sloggett A, McPherson K. Mortality among appropriately referred patients refused admission to intensive care units. Lancet. 1997; 350(9070):7-11.

10. Joynt GM, Gomersall CD, Tan P, Lee A, Cheng CA, Wong EL. Prospective evaluation of patients refused admission to an intensive care unit: Triage, futility, and outcome. Intensive Care Med. 2001; 27(9):1459-65.

11. Augustin M, Price L, Andoh-Duku A, LaCamera P. Impact of Delayed Admission to the Intensive Care Unit from the Emergency Department upon Sepsis Outcomes and Sepsis Protocol Compliance. Crit Care Res Pract. 2017; 2017:1-7.
12. Rivers E, Nguyen B, Havstad S, Ressler J, Muzzin A, Knoblich B, et al. Early goal-directed therapy in the treatment of severe sepsis and septic shock. N Engl J Med. 2001; 345:1368-77.

13. Associação de Medicina Intensiva Brasileira (AMIB)(BR). Censo AMIB 2016. Disponível em: http://www.amib.org. br/censo-amib/censo-amib-2016/

14. Brasil - Associação de Medicina Intensiva Brasileira (AMIB)(BR). Dados atualizados sobre leitos de UTI no Brasil. 2020. Disponível em: https://www.amib.org.br/ fileadmin/user_upload/amib/2020/abril/28/dados_uti_ amib.pdf

15. Ramos MC, Cruz LP, Kishima VC, Pollara WM, Lira ACO, Couttolenc BF. Performance evaluation of hospitals that provide care in the public health system, Brazil. Rev Saúde Pública. 2015; 49: 43.

16. Sprivulis PC, Silva JA, Jacobs IG, Frazer ARL, Jelinek $\mathrm{GA}$. The association between hospital overcrowding and mortality among patients admitted via Western Australian emergency departments. Med J Aust. 2006;184 (5): 208-212.

\section{Conflito de interesses}

Os autores declaram não haver qualquer conflito de interesses no presente artigo.

Autor para correspondência:

Patricia Tessari

patriciatessari@hotmail.com

Editor:

Prof. Dr Felipe Villela Gomes

Recebido em: 04/05/2020

Aprovado em: 09/11/2020 